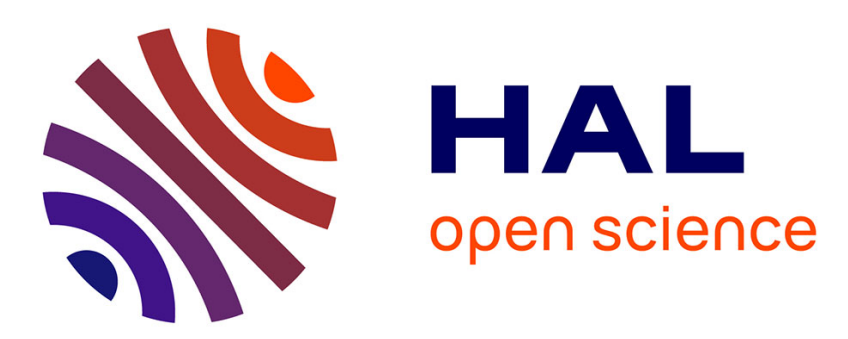

\title{
Temperature effects on the generation of steepened waves by supersonic temporal round jets \\ Pierre Pineau, Christophe Bogey
}

\section{To cite this version:}

Pierre Pineau, Christophe Bogey. Temperature effects on the generation of steepened waves by supersonic temporal round jets. 25th AIAA/CEAS Aeroacoustics Conference, May 2019, Delft, Netherlands. 10.2514/6.2019-2707 . hal-02334580

\section{HAL Id: hal-02334580 \\ https://hal.science/hal-02334580}

Submitted on 26 Nov 2020

HAL is a multi-disciplinary open access archive for the deposit and dissemination of scientific research documents, whether they are published or not. The documents may come from teaching and research institutions in France or abroad, or from public or private research centers.
L'archive ouverte pluridisciplinaire HAL, est destinée au dépôt et à la diffusion de documents scientifiques de niveau recherche, publiés ou non, émanant des établissements d'enseignement et de recherche français ou étrangers, des laboratoires publics ou privés. 


\title{
Temperature effects on the generation of steepened waves by supersonic temporal round jets
}

\author{
Pierre Pineau * and Christophe Bogey ${ }^{\dagger}$ \\ Univ Lyon, École Centrale de Lyon \\ LMFA, UMR CNRS 5509 \\ 69134 Écully, France
}

\begin{abstract}
Numerical simulations of temporally-developing round jets at a diameter-based Reynolds number of 12,500 are carried out with the aim of investigating temperature effects on the formation of the steepened acoustic waves usually associated with crackle noise. One isothermal and four hot jets at a static temperature equal to 2 or 4 times that of the ambient medium are considered. The isothermal jet has a Mach number of 2 while the hot jets have either the same Mach number or the same jet speed as the isothermal jet. At a constant Mach number, the pressure levels and skewness factors are higher at a higher temperature, indicating a more pronounced steepened aspect of the acoustic waves. This increase is due to the rise of the jet speed from 2 to 4 times the ambient sound speed in that case. When the jet velocity is constant, lower pressure levels are obtained at a higher temperature, and a slight reduction of the skewness factor is observed. This reduction is explained by the decrease of the ratio between the convection speed and the jet velocity with temperature. The present study thus allows us to isolate the effects of temperature on the generation of steepened waves near high-speed free shear flows.
\end{abstract}

\section{Introduction}

The effects of temperature on high-speed supersonic jet noise have been the topic of many investigations over the last decades [1-5]. In particular, the influence of this parameter on Mach wave radiation has been studied by Seiner at al. [3], Kearney-Fisher et al. [6], and Greska et al. [7], among others. Mach waves are generated when the convection speed $u_{c}$ of coherent structures inside the jet is higher than the ambient sound speed $a_{\infty}$. In such a case, straight, elongated wavefronts are emitted and propagate in the downstream direction at the angle $\alpha$ given by

$$
\cos \alpha=\frac{a_{\infty}}{u_{c}}
$$

When the temperature increases at a constant Mach number $M_{j}=u_{j} / a_{j}$, where $u_{j}$ is the jet speed and $a_{j}$ the speed of sound, higher pressure levels are radiated by the jets and the peak of noise emission is shifted toward higher angles relative to the flow direction, as shown by Seiner et al. [3]. These effects can be explained by the rise of the jet speed. Indeed, no such effects are observed when the temperature increases for a constant jet velocity, as in the studies of Tanna et al. [1, 2]. In that case, the pressure levels decrease with temperature for all frequencies, which can be explained by the lower densities of the jets at a higher temperature.

One particular aspect of supersonic jet noise whose dependence on temperature is currently not well understood is the generation of steepened wavefronts in the near acoustic field. The formation of these waves have been reported in the very near acoustic field in experimental measurements [8, 9], as well as in numerical simulations [10 12]. When they propagate to the far field, these waves are expected to play a key role in the formation of crackle noise [13], an unpleasant perception effect. Since the inclination angles of steepened wavefronts are very close to that of Mach waves, it has been proposed that they constitute a particular, nonlinear case of this phenomenom [12-14]. The effects of temperature on their formation have been studied by Krothapalli et al. [4], who performed optical visualizations of supersonic jets at Mach 2 and at total temperatures ranging from $580 \mathrm{~K}$ to $1250 \mathrm{~K}$. They observed an increase of the strength and frequency of occurrence of the waves associated with crackle with temperature. Mora et al. [8] also carried out measurements in the near acoustic field of hot and cold supersonic jets at $M_{j}=1.5$. At a total temperature of $600 \mathrm{~K}$, the skewness and

\footnotetext{
* PhD, now post-doctoral fellow at CNES, pierre.pineau @ doctorant.ec-lyon.fr

$\dagger$ CNRS Research Scientist, AIAA Senior Member \& Associate Fellow, christophe.bogey@ec-lyon.fr
} 
kurtosis factors of pressure fluctuations and their time-derivative are higher than for the cold jet, indicating the presence of waves with a more steepened aspect at a higher temperature. However, since the jets considered in these studies have different exhaust velocities, it is not entirely clear whether this result is a direct consequence of the temperature increase, or if it is due to the higher velocities of the jets at a higher temperature. Very few studies have attempted to characterize the influence of temperature on the formation of steepened waves at a constant jet speed. One exception is the work of Nichols et al. [15], who performed numerical simulations of two hot jets at an acoustic Mach number of 2.7 and at static temperature ratios $T_{j} / T_{\infty}$ of 2.46 and 3.03. In these simulations, the skewness factor of the pressure fluctuations obtained for the hottest jet is slightly higher than for the jet at $T_{j} / T_{\infty}=2.46$. However, given the proximity of the two temperature ratios considered, further investigations are needed to conclude on the precise role of temperature in this case.

In the present study, the effects of temperature on shock formation near the jets are investigated. More particularly, the main objective is to determine to what extent the steepened aspect of the waves is influenced by a temperature rise at a constant speed, in order to isolate the effects of temperature alone on their formation process. To this end, simulations of isothermal and hot temporal round jets are carried out. Temporal jets are used here as simplified models for the generation of sound by high-speed axisymmetric jets. In the past, such temporal simulations have been used to investigate sound generation mechanisms in subsonic [16-18], as well as in supersonic [11, 19,-21] free-shear flows. The five jets are at a Reynolds number of 12,500. The reference jet is isothermal and has a Mach number of 2 and its flow and sound fields are compared with those of four hot jets at static temperatures of $2 T_{\infty}$ and $4 T_{\infty}$. Two jets are considered for each temperature. The first jet is at a Mach number of 2, identical to that of the isothermal one, whereas the second one has the same velocity, hence an acoustic Mach number of 2. These two sets of simulations allow us to investigate the effects of a temperature rise when the jet speed varies or remains constant. This approach, we expect, will help us to clarify the precise role of temperature in the mechanisms involved in shock formation.

The paper is organized as follows. First the different jet parameters are introduced and the numerical procedure used for their computations are presented. The temporal development of the jets is then described by means of snapshots and statistical results computed over the azimuthal and axial directions. The influence of temperature on the sound waves generated during the jets development is then described by considering the variations with temperature of the pressure skewness. Finally, the variations with temperature of the pressure skewness are related to those of the convection speeds inside the jet with the aim to isolate the mechanisms by which a temperature rise can affect shock formation.

\section{Numerical methodology}

\section{A. Jet paramaters}

Five simulations of temporally-developing jets are performed in this study. The main jet parameters are given in Table 1. Three of the jets have a Mach number $M_{j}=u_{j} / a_{j}$ of 2, where $u_{j}$ is the jet velocity and $a_{j}$ is the speed of sound. The static temperature $T_{j}$ of these jets, which are labeled M20T1, M20T2 and M20T4, is equal to 1, 2, and 4 times the ambient temperature $T_{\infty}=293 \mathrm{~K}$. While the isothermal jet constitutes the reference case of this study, the jets M20T2 and M20T4 allow us to characterize the effects of a temperature rise at a constant Mach number. In that case, the jet speed $u_{j}$ increases with the temperature, leading to acoustic Mach numbers $M_{a}=u_{j} / a_{\infty}$ of 2.8 for M20T2 and 4 for M20T4, where $a_{\infty}$ is the speed of sound in the ambient medium. In order to isolate the effects of temperature, it can also be useful to investigate the effects of a temperature rise at a constant jet speed. This is done here by simulating two hot jets at $T_{j}=2 T_{\infty}$ and $4 T_{\infty}$ at the same speed as the reference isothermal jet, leading to an acoustic Mach number of 2 . Since the speed of sound increases with temperature, the Mach number of those jets, labeled M14T2 and M10T4, are 1.4 and 1 , respectively. It is noteworthy that the jet density $\rho_{j}$ decreases with the temperature, according to $\rho_{j}=p_{\infty} /\left(r T_{j}\right)$, where $p_{\infty}$ is the ambient pressure and $r=287 \mathrm{~J} \cdot \mathrm{K}^{-1} \cdot \mathrm{kg}^{-1}$ is the specific ideal gas constant. As a result, while the density of the isothermal jet is equal to that of the ambient medium, those of the hot jets are lower: namely $\rho_{j}=0.5 \rho_{\infty}$ for $\mathrm{M} 20 \mathrm{~T} 2$ and M14T2, and $\rho_{j}=0.25 \rho_{\infty}$ for M20T4 and M10T4. The different speeds and densities of the five present jets lead to different values for the convective Mach number defined, following Papamoschou \& Roshko [22], as

$$
M_{c}=\frac{M_{j}}{1+a_{\infty} / a_{j}} .
$$

The convective Mach number, which reveals the importance of compressibility on the development of the mixing layers, is equal to 0.67 for M10T4, 0.82 for M14T2, 1 for M20T1, 1.17 for M20T2 and 1.33 for M20T4. Therefore, 
Table 1 Parameters for the isothermal and hot jets.

\begin{tabular}{cccccccccc}
\hline Jet & $T_{j} / T_{\infty}$ & $M_{j}$ & $M_{a}$ & $M_{c}$ & $R e_{D}$ & $\delta_{\theta} / r_{0}$ & $u_{z}^{\prime}(t=0) / u_{j}$ & $t_{f} u_{j} / r_{0}$ & $n_{\mathrm{run}}$ \\
\hline M20T1 & 1 & 2 & 2 & 1 & 12500 & 0.018 & 0.02 & 70 & 4 \\
\hline M20T2 & 2 & 2 & 2.8 & 1.17 & 12500 & 0.018 & 0.02 & 100 & 3 \\
M20T4 & 4 & 2 & 4 & 1.33 & 12500 & 0.018 & 0.02 & 100 & 3 \\
\hline M14T2 & 2 & 1.4 & 2 & 0.82 & 12500 & 0.018 & 0.02 & 60 & 3 \\
M10T4 & 4 & 1 & 2 & 0.67 & 12500 & 0.018 & 0.02 & 60 & 3 \\
\hline
\end{tabular}

compressibility effects are expected to be stronger with increasing temperature when $M_{j}=2$, but to weaken when $M_{a}=2$.

The diameter-based Reynolds number is defined as $\operatorname{Re}_{D}=u_{j} D / v_{j}$, where $D=2 r_{0}$ is the initial jet diameter, and $v_{j}$ is the centerline kinematic viscosity. Since the jets have different temperature ratios, the latter is computed from $T_{j}$ using the Sutherland law

$$
v_{j}=v_{0}\left(\frac{T_{j}}{T_{0}}\right)^{5 / 2} \frac{T_{0}+S}{T_{j}+S},
$$

where $v_{0}=1.345 \times 10^{-5} \mathrm{~m}^{2} \cdot \mathrm{s}^{-1}, T_{0}=273 \mathrm{~K}$, and $S=111 \mathrm{~K}$. At initial time, the velocity field is initialized by the hyperbolic tangent profile represented in figure 1 (a). The shear-layer momentum thickness $\delta_{\theta}$ of this profile is chosen such that $\delta_{\theta} / r_{0}=2 / \sqrt{R e_{D}}=0.018 r_{0}$, following an empirical relation obtained for initially-laminar subsonic jets [23]. The initial temperature profiles are determined from the Crocco-Busemann relation

$$
\frac{T}{T_{j}}=\frac{T_{\infty}}{T_{j}}-\left(\frac{T_{\infty}}{T_{j}}-1\right) \frac{u_{z}}{u_{j}}+\frac{\gamma-1}{2}\left(\frac{u_{j}}{a_{j}}\right)^{2} \frac{u_{z}}{u_{j}}\left(1-\frac{u_{z}}{u_{j}}\right),
$$

and are plotted in figure 1 b) for M20T1, M20T2 and M20T4. For M20T2 and M20T4, the temperature is constant in the jet core and decreases down to $T_{\infty}$ away from the flow. For the isothermal jet, a slight temperature excess is visible at around $r=r_{0}$. It is caused by the heating of the shear layers because of compressibility effects, as derived from relation (4). Finally, low-amplitude velocity perturbations are added at $t=0$ inside the jet shear layers in order to favor the flow transition from a laminar to a turbulent state. They are vortex rings of random azimuthal mode, phase and amplitude as proposed in Bogey [18]. Their mean amplitude has been tuned so that the initial turbulence rate is of $2 \%$. As a result, the shear layers of all jets are in a weakly disturbed state at $t=0$.

\section{B. Numerical methods}

The simulations are very well-resolved Large-Eddy Simulations (LES) performed by solving the compressible Navier-Stokes equations in cylindrical coordinates $(r, \theta, z)$ using high-order finite differences. In the computations, the spatial derivatives are evaluated using a centered fourth-order eleven-point low-dispersion finite-difference scheme [24], and time integration is carried out using a second-order, six-stage Runge-Kutta algorithm [24]. Near the jet axis, the method proposed by Mohseni \& Colonius [25] is applied to avoid singularity at $r=0$. As a result, the first discretization point is placed at a distance $r=\Delta r / 2$ from the jet axis, where $\Delta r$ is the radial mesh spacing close to the jet axis. The azimuthal derivatives are also evaluated using fewer points than permitted by the grid, in order to reduce the constraint on the time-step arising from the use of cylindrical coordinates near the centerline [26]. More precisely, the effective azimuthal resolution is 16 for the row of points nearest to the jet axis. It progressively increases with the radial distance, and reaches $n_{\theta}=256$ for $r=0.25 r_{0}$. At the end of each time step, a standard, twelfth-order selective filter is applied to remove grid-to-grid oscillations. Another role of the filter is to relax the subgrid-scale energy near the grid cut-off wavenumber. A shock-capturing scheme [27] based on the application of an adaptive filtering procedure is also applied to damp Gibbs oscillations around shocks. Finally, the radiation conditions of Tam \& Dong [28] are applied at the radial boundary of the computational domain in order to allow the acoustic waves to leave the computational domain without causing significant spurious reflexions. At the axial inflow and outflow, periodicity conditions are imposed. 
(a)

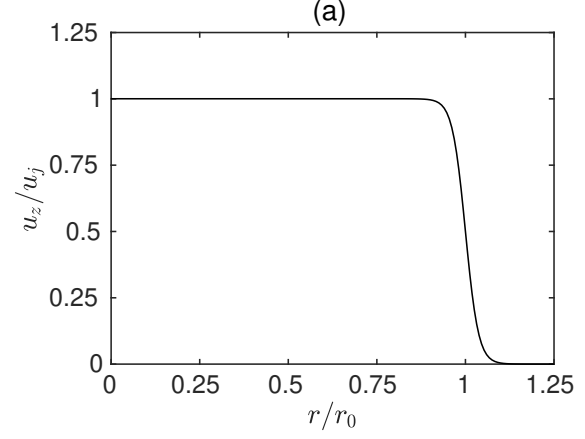

(b)

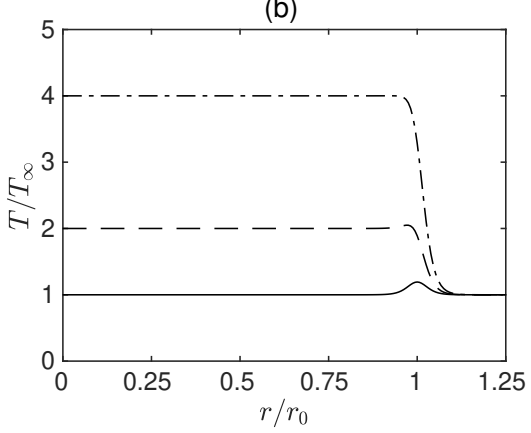

Fig. 1 Initial radial profiles of (a) axial velocity, and (b) static temperature for $M_{j}=2$ and $-T_{j} / T_{\infty}=1$, $---T_{j} / T_{\infty}=2$, and $-\cdot-\cdot T_{j} / T_{\infty}=4$.

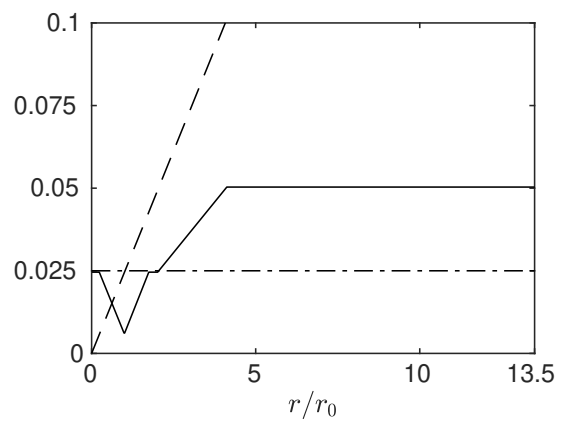

Fig. 2 Radial profiles of axial, azimuthal, and radial mesh spacings; $-\Delta z / r_{0}$.

$\Delta r / r_{0},--r \Delta \theta / r_{0}$ and -

\section{Computational parameters}

The mesh used for the five simulations extends over a distance of $240 r_{0}$ in the axial direction, and out to $13 r_{0}$ in the radial direction. It contains $n_{r} \times n_{\theta} \times n_{z}=382 \times 256 \times 9600$ points, yielding a total number of 940 millions points. The radial variations of the radial, azimuthal and axial mesh spacing are plotted in figure 2 The axial spacing $\Delta z$ is constant and equal to $\Delta z=0.025 r_{0}$, whereas the radial spacing varies in the radial direction. It is such that $\Delta r=\Delta z$ on the jet axis, and reaches a minimum of $\Delta r=0.0063 r_{0}$ at $r=r_{0}$. It then progressively increases up to $r=4 r_{0}$, after which it remains equal to $0.05 r_{0}$. This spacing corresponds to a Strouhal number of 5 for an acoustic wave discretized by four points per wavelength. The simulations are carried out until a simulation time of $t_{f}=70 r_{0} / u_{j}$ for M20T1, 60 $r_{0} / u_{j}$ for M14T2 and M10T4, and 100 $r_{0} / u_{j}$ for M20T2 and M20T4. They necessitate approximately 10,000 time steps, which requires 10,000 CPU hours. In all cases, different runs of the same jet are performed by changing the values of the random seeds used in the excitation procedure. The number $n_{\text {run }}$ of computations performed for a given jet is equal to 4 in the case of the isothermal jet, and to 3 for the hot jets.

\section{Results}

\section{A. Snapshots}

Snapshots of vorticity norm and static temperature obtained for M20T2 are given in figure 3 at $t=10 r_{0} / u_{j}, 20 r_{0} / u_{j}$, $30 r_{0} / u_{j}$ and $40 r_{0} / u_{j}$. At $t=10 r_{0} / u_{j}$, the jet core is surrounded by thin mixing layers of vortical flow, in which slight perturbations are visible, indicating the presence of instability waves. The temperature field is uniform inside the jet core, but weak fluctuations are present at the interface between the flow and the ambient medium. At $t=20 r_{0} / u_{j}$, the mixing layers are thicker than previously. More fine-scale turbulence appear in the vorticity field, because of the mixing-layer transition from a laminar to a turbulent state. This is also discernible in the temperature field, where turbulent structures are distinguishable at the jet boundary. At $t=30 r_{0} / u_{j}$ the mixing layers join and merge on the jet axis. Simultaneously, a decrease of the temperature inside the jet core is noticeable in figure 3 (c) and (d), due to the 
(a)
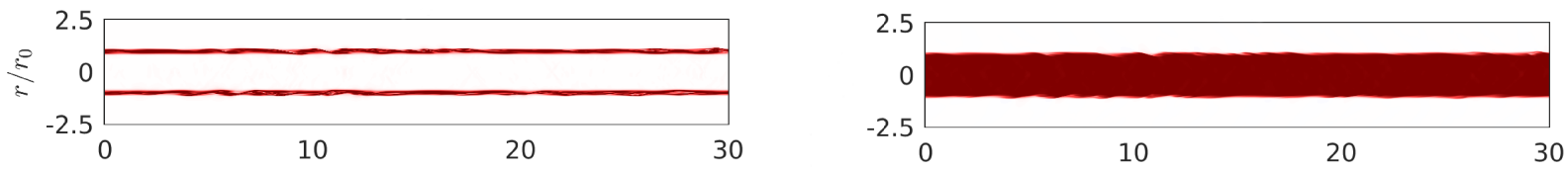

(b)
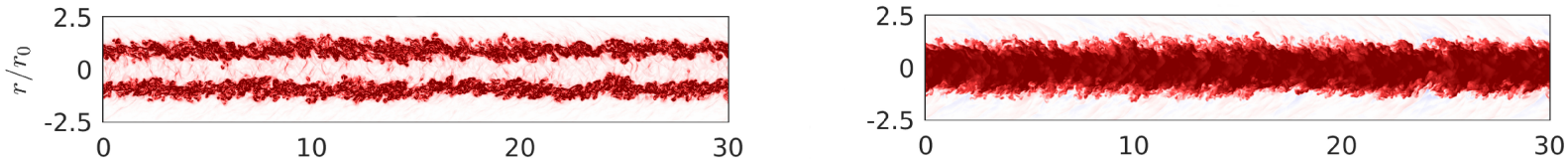

(c)
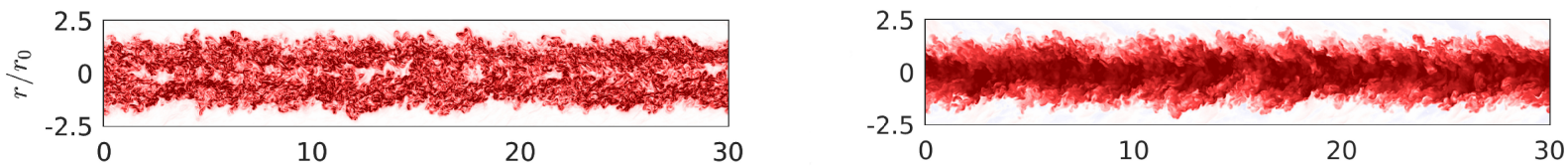

(d)
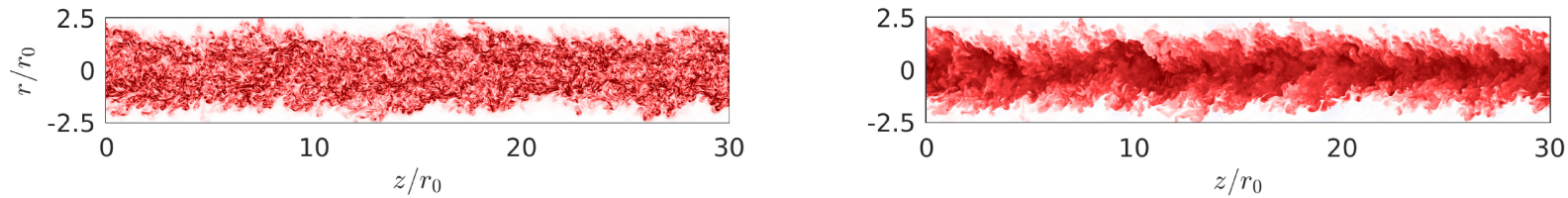

Fig. 3 Snapshots of vorticity norm (left) and static temperature (right) for M20T2 at (a) $t=10 r_{0} / u_{j},(b)$ $20 r_{0} / u_{j}$, (c) $30 r_{0} / u_{j}$, and (d) $40 r_{0} / u_{j}$. The color scales range up to $4 u_{j} / r_{0}$ for the vorticity, and from $T_{\infty}$ to $T_{j}=2 T_{\infty}$ for temperature, from white to red.

mixing between the hot gases inside the jet core and the cold ambient air.

\section{B. Mean flow}

The time variations of the mean axial velocity are shown in figure 4 (a). For all jets, the velocity is constant and equal to its initial value $u_{j}$ at early times and remains so as long as the centerline flow is irrotational. It rapidly decreases afterwards, as low-speed gas is entrained inside the jet core after the merging of the shear layers. The core-closing time $t_{c}$ is defined as the time when the centerline mean velocity $\left\langle u_{z \text {, axis }}\right\rangle$ is such that $\left\langle u_{z}\right.$,axis $\rangle=0.95 u_{j}$. When $M_{a}=2$, it is equal to $25.3 r_{0} / u_{j}$ for M20T1, to $20.2 r_{0} / u_{j}$ for M14T2, and to $17.0 r_{0} / u_{j}$ for M10T4. It is thus lower at a higher temperature, which can be explained by the decrease of the jet density, but also by compressibility effects. Indeed, as reported in Table 1, the convective Mach number $M_{c}$ is lower at a higher temperature, leading to a faster spreading of the jet shear layers [22, 29]. The variations of the core-closing time are less pronounced when the Mach number $M_{j}$ is equal to 2 . In this case, the potential core closes at $t_{c}=25.3 r_{0} / u_{j}$ for M20T1, at $26.2 r_{0} / u_{j}$ for M20T2, and at 29.8 $r_{0} / u_{j}$ for M20T4. It is thus slightly longer at a higher temperature. This can be explained by the increase of the convective Mach number with temperature when $M_{j}=2$, which leads to a slower spreading of the jet shear layers, delaying their merging on the jet axis.

The maximum of the root-mean-square values of axial velocity fluctuations is represented in figure 4 (b) as a function of $\left(t-t_{c}\right) u_{j} / r_{0}$. In all cases, a peak value of approximately $0.2 u_{j}$ is reached at $t \simeq t_{c}-12 r_{0} / u_{j}$. Following Bogey $\&$ Bailly [30], this peak can be attributed to pairings between initial vortical structures in the shear layers as the jets transition from a laminar to a turbulent state. After this peak, the velocity fluctuations gradually decrease as the jets spread. Once the jets have transitioned to a turbulent state, it can also be noted that the turbulence intensities collapse well, which highlights the relevance of the core-closing time $t_{c}$ as a characteristic time scale of the flow development. Consequently, this parameter will be used throughout the rest of this paper to perform comparison between the different jet flows. 
(a)

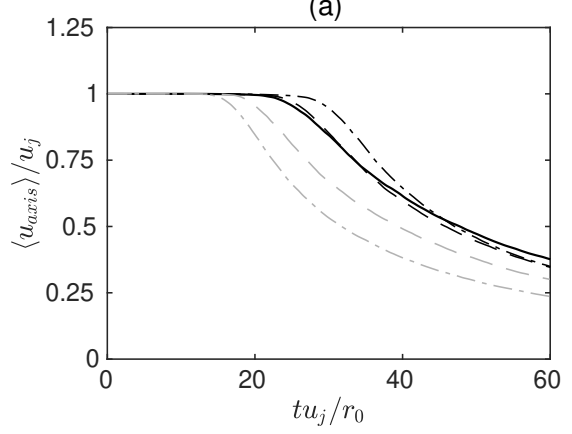

(b)

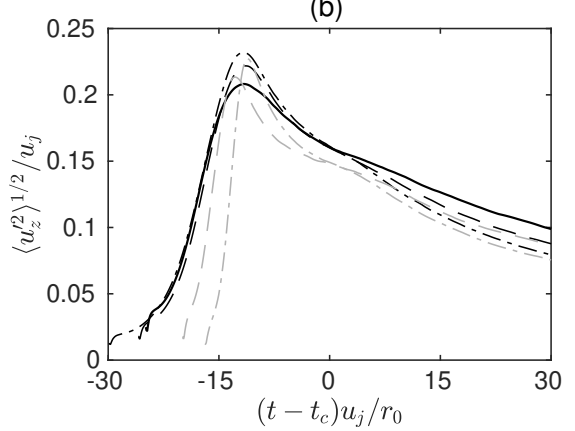

Fig. 4 Time variations of (a) mean centerline velocity and (b) peak turbulence intensities for M20T1, - - M20T2, - - - M20T4, - - M14T2 and $-\cdot-\cdot$ M10T4.

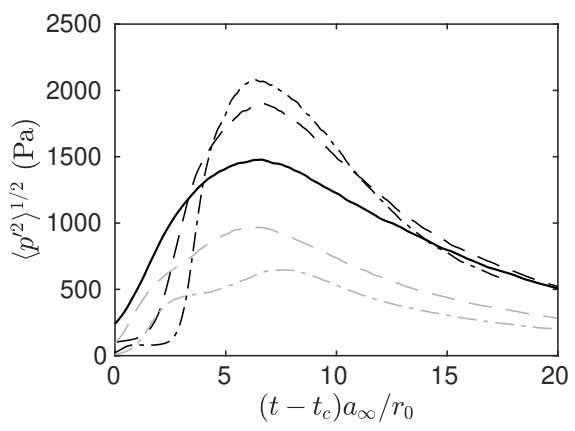

Fig. 5 Time variations of the RMS values of pressure fluctuations at $r=8 r_{0}$ for - M20T1, - M20T2, - - . M20T4, - - M14T2 and - - M10T4.

\section{Near acoustic field}

\section{Sound pressure levels and snapshots}

The time variations of the pressure levels computed at a distance $r=8 r_{0}$ from the jet axis are plotted in figure 5 as a function of $\left(t-t_{c}\right) a_{\infty} / r_{0}$. For all jets, a peak is reached after the core closing time at approximately $t=t_{c}+7 r_{0} / a_{\infty}$. The peak of pressure levels is of $1500 \mathrm{~Pa}$ for M20T1, 970 Pa for M14T2 and 650 Pa for M10T4. It thus decreases with the temperature when the jet speed is constant, in agreement with the experimental results of Tanna et al. [2]. This decrease can be explained by the lower densities of the hot jets at a higher temperature, leading to a weakening of the sound sources [2]. A different trend is observed when the jet temperature is raised at a constant Mach number. In that case, the peak levels are of $1900 \mathrm{~Pa}$ for M20T2 and $2100 \mathrm{~Pa}$ for M20T4, and is higher at a higher temperature. This increase is consistent with the results of Seiner et al. [3] for spatially-developing Mach 2 jets. For these jets, the rise of the jet velocity from $u_{j}=2 a_{\infty}$ to $4 a_{\infty}$, compensates the weakening of the sound sources caused by the decrease of the jet density.

Snapshots of pressure fluctuations and vorticity norm obtained at $t=t_{c}+7 r_{0} / a_{\infty}$ for the isothermal and hot jets are represented in figure 6 for the isothermal jet M20T1 as well as for the two jets M20T4 and M10T4 at $T_{j}=4 T_{\infty}$. In the $(r, z)$ plane, straight, inclined wavefronts, whose associated wavelengths are of the order of the jet diameter are visible near the jets. The presence of these wavefronts, combined with jet velocities that are well above the ambient sound speed, strongly suggest that both the isothermal and hot jets radiate Mach waves. Moreover, a strong azimuthal correlation of the radiated pressure field appears in all cases in the visualizations of the $(r, \theta)$ plane, which is also typical of Mach wave radiation [31]. Despite these similarities, some properties of the radiated acoustic waves differ. Notably, the waves radiated by the hot jet M20T4 are more inclined than those produced by the isothermal jet M20T1, which can be explained by the higher speed of M20T4. As a result, convection speeds inside the jet are higher, leading to a shift of the sound field directivity toward higher angles, following relation (1). No such effect is observed in figure 6 (c) for the jet M10T4, which have the same velocity as the isothermal jet M20T1. On the contrary, the sound waves radiated by this jet are slightly less inclined than for M20T1 and have, to some extent, a curved aspect. Finally, it can also be noted 
(a)

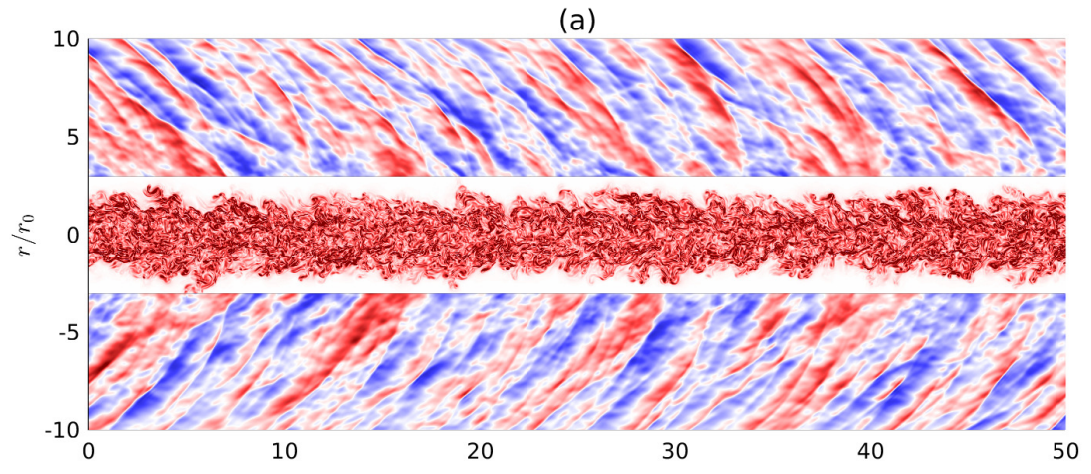

(b)

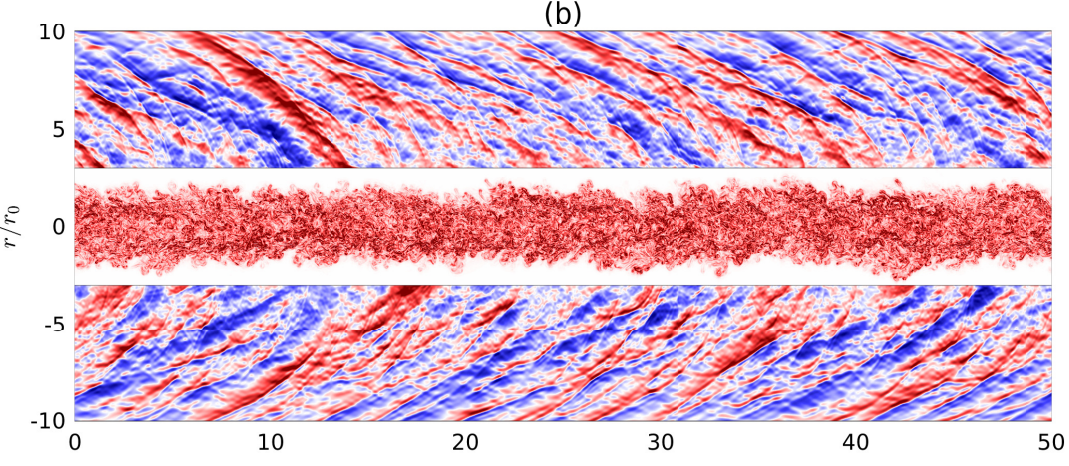

(c)

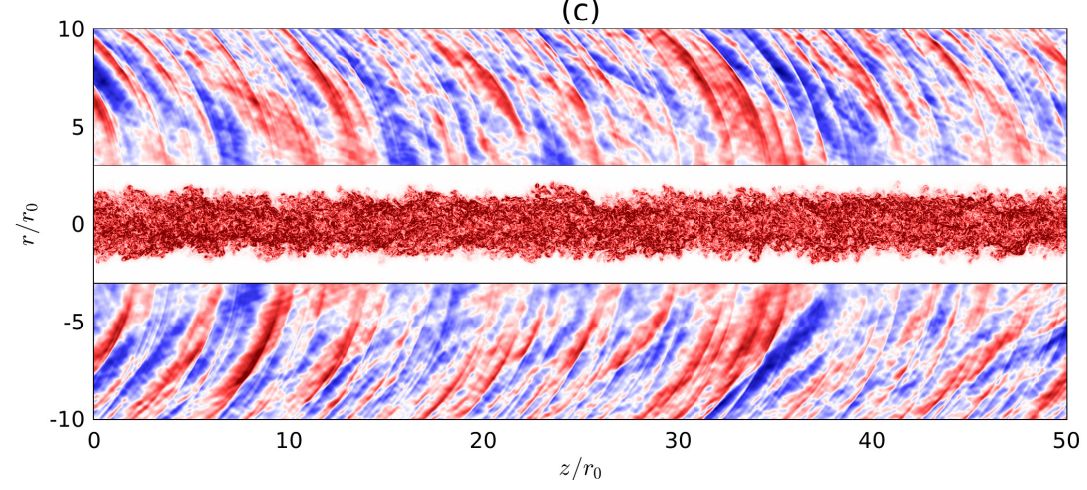

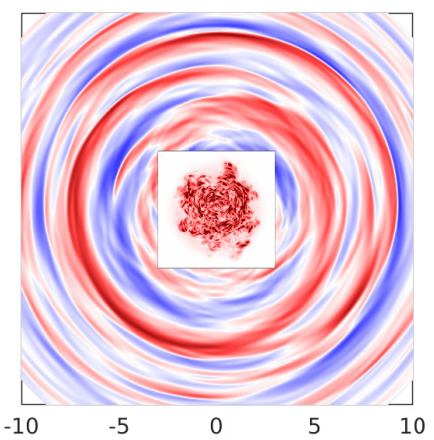
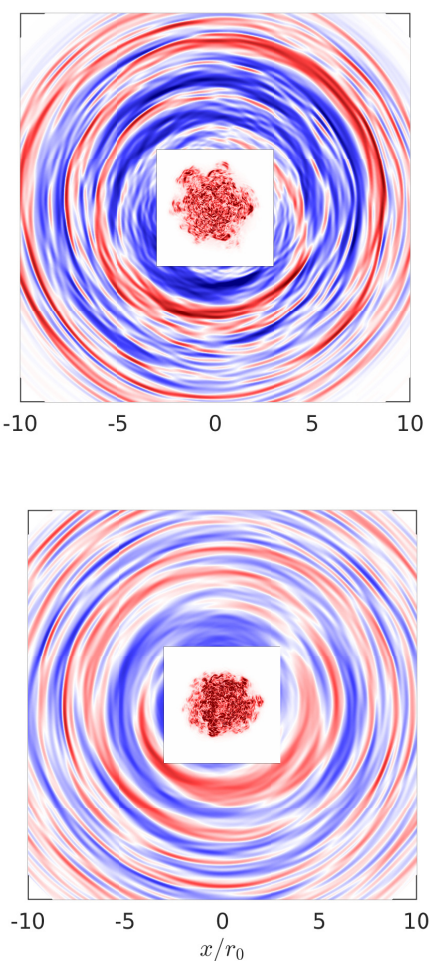

Fig. 6 Snapshots of pressure fluctuations and vorticity norm at $t=t_{c}+7 r_{0} / a_{\infty}$ for the jets (a) M20T1, (b) M20T4 and (c) M10T4. The color scales range (a,b) from -6000 to $6000 \mathrm{~Pa}$ and (c) -4000 to $4000 \mathrm{~Pa}$ for the pressure fluctuations, and up to $3 u_{j} / r_{0}$ for the vorticity, from blue to red.

that shock waves are present in the jet near fields. This is more clearly visible for the jet M20T4 in figure 6(b), for which $M_{a}=4$, than near the jets M20T1 and M10T4, at $M_{a}=2$. This suggests that the steepened aspect of the acoustic waves is more pronounced when the jet speed is high, which will be investigated in what follows.

\section{Shock-like structures}

The axial variations of the pressure fluctuations obtained at $r=8 r_{0}$ at $t=t_{c}+7 r_{0} / a_{\infty}$ are plotted in figures 7 for the isothermal jet M20T1, as well as for the jets M10T4 and M20T4 at $T_{j}=4 T_{\infty}$. They are normalized by their standard deviations. For the isothermal jet in figure 7(a), strong pressure peaks emerge as, for instance, at $z=13 r_{0}$ and $z=19 r_{0}$. These peaks consist of a fast compression phase, leading to pressure levels which can be three times higher than the standard deviation, and followed by an expansion phase which is more gradual than the compression phase. The shapes of these shock-like structures are very similar to those depicted by Buchta \& Freund [11] in their simulations of temporally-developing plane mixing layers. They also remind us of those observed in the sound field of high-speed 
(a)

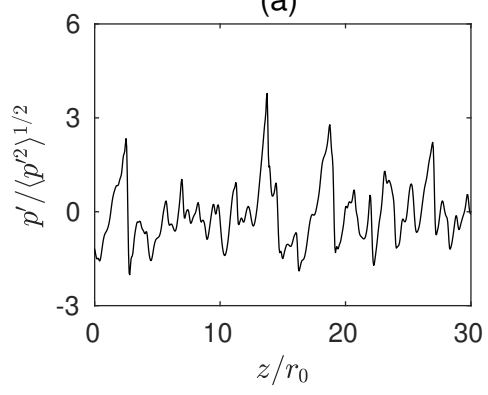

(b)

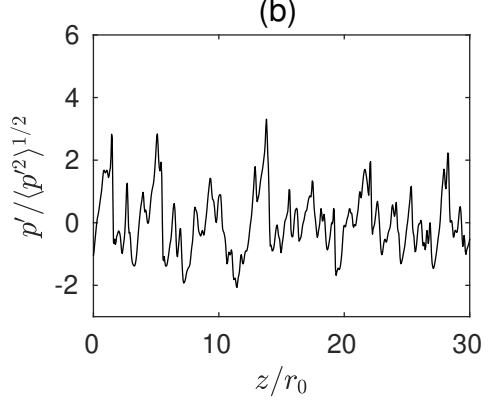

(c)

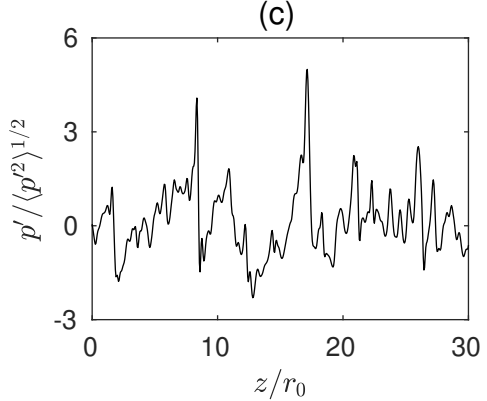

Fig. 7 Pressure fluctuations normalized by their standard deviations at $r=8 r_{0}$ and $t=t_{c}+7 r_{0} / a_{\infty}$ for (a) M20T1, (b) M10T4 and (c) M20T4.
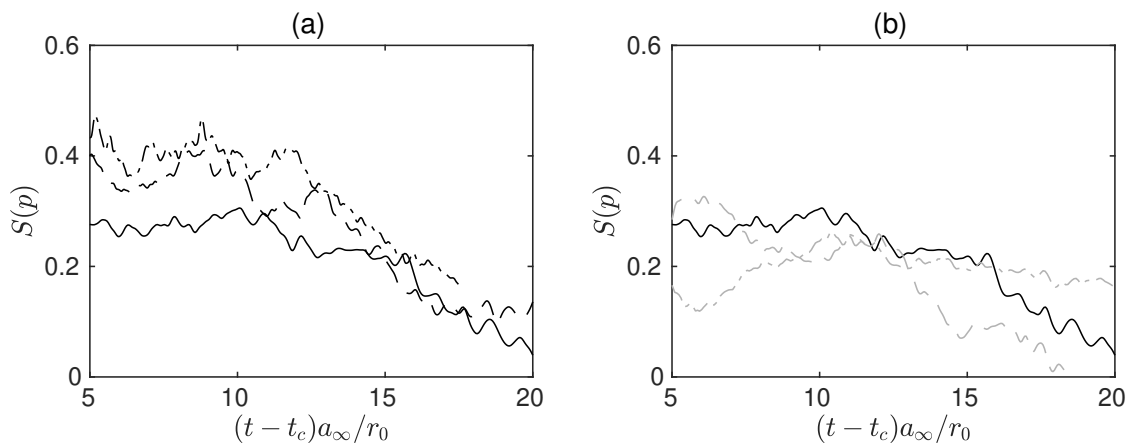

Fig. 8 Time variations of the skewness factor of pressure fluctuations at $r=8 r_{0}$ for - M20T1, - M20T2, - - . M20T4, - - M14T2 and - - M10T4.

spatially-developing supersonic jets emitting crackle noise [13, 32]. These structures are also visible in the pressure fields radiated by the hot jets, for instance at $z=13 r_{0}$ for M10T4 in figure 7(b), and at $z=8 r_{0}$ and $z=18 r_{0}$ for M20T4 in figure 7(c). For M20T4, a peak value of 5 times the standard deviation is even found at $z=18 r_{0}$, which suggests that this jet radiates more intense steepened waves than the other jets at $M_{a}=2$.

A more formal assessment of the steepened aspect of the acoustic waves radiated by the jets can be obtained by computing the skewness factor of the pressure fluctuations, defined as

$$
S(p)=\frac{\left\langle p^{\prime 3}\right\rangle}{\left\langle p^{\prime 2}\right\rangle^{3 / 2}} .
$$

Indeed, positive values of $S(p)$ indicate a positive asymmetry of the pressure signals, which has been related in the past to the presence of crackle. More precisely, it has been postulated by Ffowcs Williams et al. [13] that crackle is distinctly heard when $S(p)$ is higher than 0.4 , but not when $S(p)$ is lower than 0.3. The time variations of the skewness factors computed at $r=8 r_{0}$ are plotted in figure 8 as a function of $\left(t-t_{c}\right) a_{\infty} / r_{0}$ for the five present jets. For the jets at $M_{j}=2$, in figure $8(\mathrm{a}), S(p)$ significantly deviates from zero and is positive for $t_{c}+5 r_{0} / a_{\infty} \leq t \leq t_{c}+10 r_{0} / a_{\infty}$, when the pressure levels are at their peak. During that period $S(p)$ is approximately constant and equal to 0.27 for M20T1, 0.36 for M20T2 and 0.40 for M20T4. Consequently, at Mach 2, the skewness factor increases with temperature, which indicates that positive pressure peaks are more proeminent as the temperature, and hence the jet speed, is raised. This increase is consistent with the trends reported in previous experimental studies. In particular, Mora et al. [8] reported a rise of the skewness factor from 0.09 to 0.32 in the far acoustic field of a Mach 1.5 jet when its total temperature was increased from $300 \mathrm{~K}$ to $600 \mathrm{~K}$. Krothapalli et al. [4] also noted an increase of the intensity and the frequency of occurrence of steepened waves in the vicinity of supersonic jets at Mach 2 as the total temperature was raised from $580 \mathrm{~K}$ to $1250 \mathrm{~K}$. No such increase of the pressure skewness is however observed when the temperature rises at a constant jet speed, in figure $8 \mathrm{~b}$ ). In this case, the skewness at time of peak levels, obtained by averaging $S(p)$ from $t=t_{c}+5 r_{0} / a_{\infty}$ to $t_{c}+10 r_{0} / a_{\infty}$, is of 0.28 for M20T1, 0.27 for M14T2 and 0.18 for M10T4, and slightly decreases with temperature. 


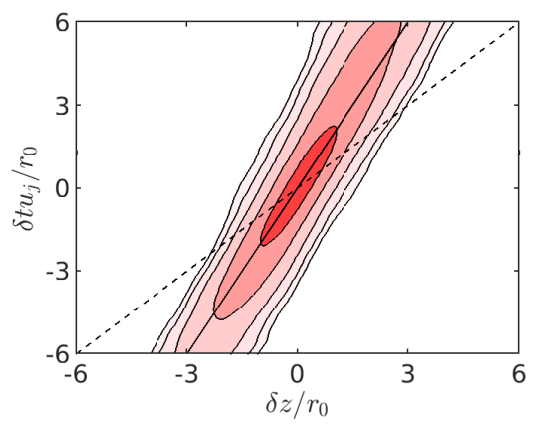

Fig. 9 Space-time autocorrelations of axial velocity fluctuations at $t=t_{c}$ and $r=r_{0}$ for M20T1. The lines indicate convection at $-a_{\infty}$ and $---u_{j}$. The color scale ranges from -1 to 1 , from blue to red.
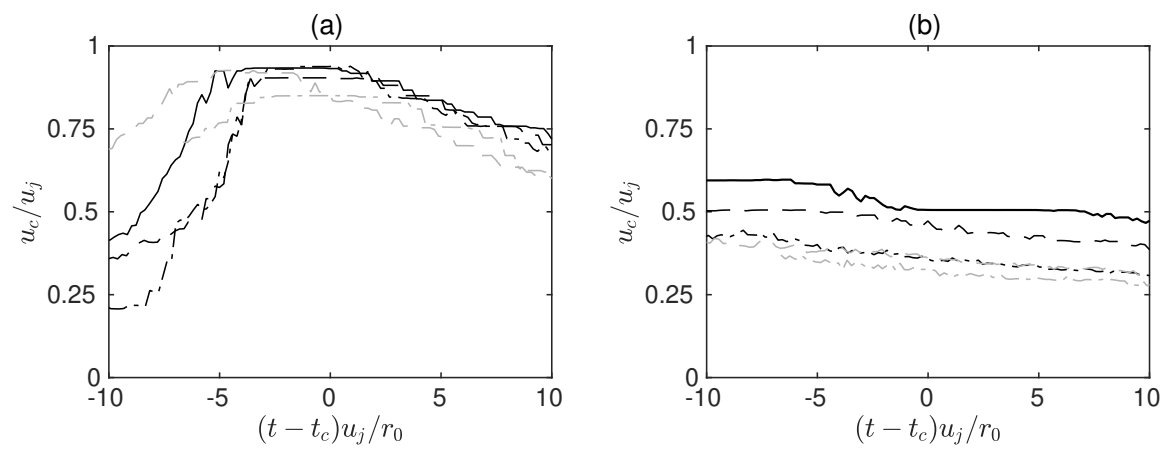

Fig. 10 Time variations of the convection speed normalized by $u_{j}$ at (a) $r=0$ and (b) $r=r_{0}$ for M20T1, - - M20T2, - - . M20T4, - - M14T2 and - - M M10T4.

\section{Convection speeds}

It has been proposed [12-14] that the generation of steepened waves in the near vicinity of supersonic jets could be viewed as an extreme, nonlinear case of Mach wave radiation by large-scale coherent structures. The convection speed of these structures have been shown to play a key role in the steepened aspect of the waves. Notably, Petitjean et al. [33] noted that supersonic convection speeds was a necessary condition for the formation of nonlinear waves in the far field, while Buchta \& Freund [11] reported an increase of the near field pressure skewness with increasing values of the convective Mach number. Consequently, in order to investigate temperature effects on the formation of steepened waves, it seems natural to investigate to what extent convection speeds inside the jets are affected by a temperature rise. To do this, the convection speeds are evaluated from the autocorrelation functions of the axial velocity fluctuations, defined as

$$
\mathcal{R}_{u_{z}^{\prime} u_{z}^{\prime}}(\delta z, \delta t)=\frac{\left\langle u_{z}^{\prime}(r, \theta, z, t) u_{z}^{\prime}(r, \theta, z+\delta z, t+\delta t)\right\rangle}{\left\langle u_{z}^{\prime 2}(r, \theta, z, t)\right\rangle^{1 / 2}},
$$

where $\delta z$ and $\delta t$ are the spatial separation and time shift, respectively. The correlation function computed at $r=r_{0}$ and at the core closing time $t_{c}$ for M20T1 is represented in figure 9 . The correlation values are maximum for $\delta z=0$ and $\delta t=0$, as expected, and remain significant along a straight elongated band. This shape indicates that flow structures are convected inside the jet while remaining coherent in time and space.

The mean convection speed of these structures can be inferred from the inclination of the correlation band. They are plotted in figure 10 at two radial locations, at $r=0$ in figure 10 (a) and at $r=r_{0}$ in figure 10 (b). Given that the velocity of the present jets varies from $2 a_{\infty}$ to $4 a_{\infty}$, the convection speeds are normalized by $u_{j}$ in order to only examine the effects of temperature. On the jet axis, the ratio between the convection velocity and the jet speed is maximum close to the core-closing time $t_{c}$, where it is slightly lower than unity. It decreases afterwards, following the decay of the mean centerline velocity. Moreover, it is slightly higher for the isothermal jet than for the hot jets. This effect of temperature on the convection speed is more clearly visible at $r=r_{0}$, where $u_{c}=0.51 u_{j}$ for M20T1, $0.47 u_{j}$ for M20T2, $0.35 u_{j}$ for 


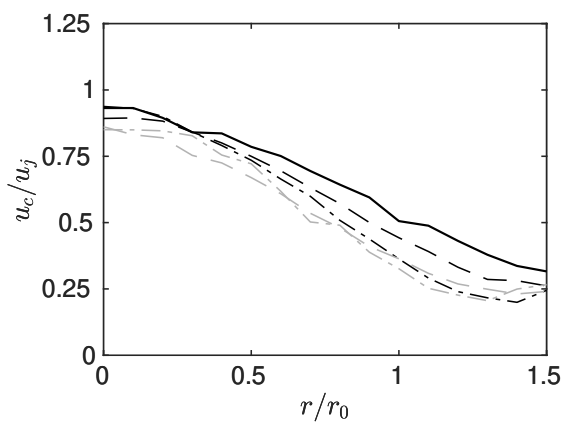

Fig. 11 Radial variations of the convection speeds at $t=t_{c}$ for - M20T1, - - M20T2, - - . M20T4, - - M14T2 and - - . M10T4.

M20T4 and M20T2 and 0.33 $u_{j}$ for M10T4. Thus, it appears that the convection speed decreases with the temperature, when normalized by the jet speed.

The radial variations of the convection speeds obtained at $t=t_{c}$ are plotted in figure 11. As expected, it is maximum close to the jet axis and decreases with the radial distance. Its decay is faster for the hot jets than for the isothermal jet, which explains why the ratio $u_{c} / u_{j}$ at $r=r_{0}$ is lower at a lower temperature in figure 10(b). The decrease of the convection speed with temperature has been observed in previous studies of hot supersonic jets [5, 34, 35]. It is also predicted by several models of the convection speed of large-scale structures in high-speed jets, including those of Papamoschou \& Roshko [22], Oertel [36], or Dimotakis [37]. Furthermore, based on the results of Buchta \& Freund [11] who highlighted the role of the convective Mach number on shock formation near the jet, lower convection speeds at a higher temperature are consistent with the decrease of the pressure skewness in figure $8 \mathrm{~b}$ ).

\section{Conclusion}

In the present study, the effects of temperature on the formation of steepened waves near high-speed supersonic jets are investigated by performing numerical simulations of temporal round jets at static temperatures equal to 1,2 and 4 times that of the ambient medium. Two of the hot jets have a Mach number of 2, identical to that of the isothermal jet, in order to investigate the effects of a temperature rise at a constant Mach number. In that case, the acoustic waves radiated during the jet development are stronger at a higher temperature and their directivity is shifted toward higher angle relative to the flow direction, as observed in corresponding experimental studies. In addition, the steepened aspect of the acoustic waves becomes more pronounced with increasing temperature, which is reflected in the rise of the pressure skewness. In these simulations, the jet speed increases from $u_{j}=2 a_{\infty}$ to $u_{j}=4 a_{\infty}$ when the temperature raises from $T_{\infty}$ to $4 T_{\infty}$. Consequently, in order to distinguish the effects of temperature from those of the jet speed, the two other hot jets considered in this study have the same velocity as the isothermal one. In that case, lower pressure levels are radiated by the jets and a slight reduction of the pressure skewness is observed at a higher temperature. The two different trends are explained by estimating the convection speeds of turbulent structures inside the jet flow. For all jets, the ratio between the convection speed and the jet speed decreases with the temperature, which could explain the decrease of the pressure skewness when the jet speed is constant. When the temperature is raised at a constant Mach number, this decrease is however compensated by the increase of the jet velocity. One shortcoming of the present analysis is that the present convection speeds, computed from single-point correlations, are not related to noise generation events. Moreover, they are dependent on the radial position, which makes it difficult to single out one parameter governing the generation of steepened waves. The present analysis could be complemented by introducing a convection speed directly related to noise generation. This could be done, for instance, by computing cross correlations between the flow and sound field, or from the computations of conditional averages, as done in a recent study of spatially-developing jets [12].

\section{Acknowledgments}

This work was granted access to the HPC resources of FLMSN (Fédération Lyonnaise de Modélisation et Sciences Numériques), partner of EQUIPEX EQUIP@MESO, and of the resources of IDRIS (Institut du Développement et des Ressources en Informatique Scientifique) under the allocation 2018-2a0204 made by GENCI (Grand Equipement National de Calcul Intensif). It was performed within the framework of the Labex CeLyA of Université de Lyon, 
operated by the French National Research Agency (Grant No. ANR-10-LABX-0060/ANR-11-IDEX-0007).

\section{References}

[1] Tanna, H. K., Dean, P. D., and Fisher, M. J., "Influence of temperature on shock-free supersonic jet noise," Journal of Sound and Vibration, Vol. 39, No. 4, 1975, pp. 429-460.

[2] Tanna, H. K., "An experimental study of jet noise. Part 1 : turbulent mixing noise," Journal of Sound and Vibration, Vol. 50, No. 3, 1977, pp. 429-444.

[3] Seiner, J., Ponton, B., M. K. Jansen, and Lagen, N., "The effects ot temperature on supersonic jet noise emission," In 14th DGLR/AIAA Aeroacoustics Conference, Vol. 1, 1992, pp. 295-307.

[4] Krothapalli, A., Venkatakrishnan, L., and Lourenco, L., "Crackle: A dominant component of supersonic jet mixing noise," AIAA paper 2000-2024, 2000.

[5] Liu, J., Corrigan, A., Kailasanath, K., and Gutmark, E., "Effects of temperature on noise generation in supersonic jets," AIAA paper 2016-2937, 2016.

[6] Kearney-Fischer, M., Kim, J. H., and Samimy, M., "A study of Mach wave radiation using active control," Journal of Fluid Mechanics, Vol. 681, 2011, pp. 261-292.

[7] Greska, B., Krothapalli, A., Horne, W. C., and Burnside, N., "A near-field study of high temperature supersonic jets," AIAA paper 2008-3026, 2008.

[8] Mora, P., Heeb, N., Kastner, J., Gutmark, E. J., and Kailasanath, K., "Impact of heat on the pressure skewness and kurtosis in supersonic jets," AIAA Journal, Vol. 52, No. 4, 2014.

[9] Fiévet, R., Tinney, C. E., Baars, W. J., and Hamilton, M. F., "Coalescence in the sound field of a laboratory-scale supersonic jet," AIAA Journal, Vol. 54, No. 1, 2016, pp. 254-265.

[10] Nichols, J. W., Lele, S. K., Ham, F. E., Martens, S., and Spyropoulos, J. T., "Crackle noise in heated supersonic jets," Journal of Engineering for Gas Turbines and Power, Vol. 135, No. 5, 2013.

[11] Buchta, D. A., and Freund, J. B., "The near-field pressure radiated by planar high-speed free-shear-flow turbulence," Journal of Fluid Mechanics, Vol. 832, 2017, p. 383-408.

[12] Pineau, P., and Bogey, C., "Study of the generation of shocks by high-speed jets using conditional averaging," AIAA Paper 2018-3305, 2018.

[13] Ffowcs Williams, J. E., Simson, J., and Virchis, V. J., "'Crackle': an annoying component of jet noise,” Journal of Fluid Mechanics, Vol. 71, 1975, pp. 251-271.

[14] Murray, N. E., and Lyons, G. W., “On the convection velocity of source events related to supersonic jet crackle," Journal of Fluid Mechanics, Vol. 793, 2016, pp. 477-503.

[15] Nichols, J. W., Lele, S. K., and Spyropoulos, J. T., “The source of crackle noise in heated supersonic jets," AIAA paper 2013-2197, 2013.

[16] Fortune, V., Lambalais, E., and Gervais, Y., "Noise radiated by a non-isothermal, temporal mixing layer. Part 1. Direct computation and prediction using compressible DNS," Theoretical and Computational Fluid Dynamics, Vol. 18, 2004 , pp. 61-81.

[17] Kleinman, R. R., and Freund, J. B., "The sound form mixing layers simulated with different ranges of turbulence scales," Physics of Fluids, Vol. 20, No. 101503, 2008, pp. 1-12.

[18] Bogey, C., "On noise generation in low Reynolds number temporal round jets at a Mach number of 0.9," Journal of Fluid Mechanics, Vol. 859, 2019, pp. 1022-1056.

[19] Terakado, D., Nonomura, T., Oyama, A., and Fujii, K., "Mach number dependence on sound sources in high Mach number turbulent mixing layers," AIAA paper 2016-3015, 2016.

[20] Pineau, P., and Bogey, C., "Numerical study of the sound fields of temporally-developing supersonic round jets," AIAA Paper 2017-3209, 2017. 
[21] Bogey, C., "On the noise generated by the potential-core closing of temporally-developing subsonic jets," AIAA Paper 2017-3851, 2017.

[22] Papamoschou, D., and Roshko, A., "The compressible turbulent shear layer : an experimental study," Journal of Fluid Mechanics, Vol. 197, 1988, pp. 453-477.

[23] Zaman, K. B. M. Q., “Effect of initial condition on subsonic jet noise,” AIAA Journal, Vol. 23, No. 9, 1985, pp. 1370-1373.

[24] Bogey, C., and Bailly, C., "A family of low dispersive and low dissipative explicit schemes for noise computation," Journal of Computational Physics, Vol. 194, No. 1, 2004, pp. 194-214.

[25] Mohseni, K., and Colonius, "Numerical treatment of polar coordinate singularities," Journal of Computational Physics, Vol. 157, No. 10, 2002, pp. 3593-3600.

[26] Bogey, C., Marsden, O., and Bailly, C., "Finite differences for coarse azimuthal discretization and for reduction of effective resolution near origin of cylindrical flow equations," Journal of Computational Physics, Vol. 230, 2011, pp. 1134-1146.

[27] Bogey, C., de Cacqueray, N., and Bailly, C., "A shock-capturing methodology based on adaptative spatial filtering for high-order non-linear computations," Journal of Computational Physics, Vol. 228, 2009, pp. 1447-1465.

[28] Tam, C. K. W., and Dong, Z., "Radiation and outflow boundary conditions for direct computation of acoustic and flow disturbances in a nonuniform mean flow," Journal of Computational Acoustics, Vol. 4, No. 2, 1996, pp. 175-201.

[29] Freund, J. B., Lele, S. K., and Moin, P., "Compressibility effects in a turbulent annular mixing layer. Part 1. Turbulence and growth rate," Journal of Fluid Mechanics, Vol. 421, 2000, pp. 229-267.

[30] Bogey, C., Marsden, O., and Bailly, C., "Influence of initial turbulence level on the flow and sound fields of a subsonic jet at a diameter-based Reynolds number of 100000,” Journal of Fluid Mechanics, Vol. 701, 2012, pp. 352-385.

[31] de Cacqueray, N., Bogey, C., and Bailly, C., "Investigation of a High-Mach-Number Overexpanded Jet Using Large-Eddy Simulation," AIAA Journal, Vol. 49, No. 10, 2011, pp. 2171-2182.

[32] Baars, W., and Tinney, C. E., "Shock-structures in the acoustic field of a Mach 3 jet with crackle," Journal of Sound and Vibration, Vol. 333, 2014, pp. 2539-2553.

[33] Petitjean, B., Viswanathan, K., and McLaughlin, D., "Acoustic pressure waveforms measured in high speed jet noise experiencing nonlinear propagation,” International Journal of Aeroacoustics, Vol. 5, No. 2, 2006, pp. 193-215.

[34] Ecker, T., Lowe, K. T., and Ng, W. F., "Eddy convection in developing heated supersonic jets," AIAA Journal, Vol. 53, No. 11, 2015, pp. 3305-3315.

[35] Gojon, R., Baier, F., Gutmark, E., and Mihaescu, M., "Temperature effects on the aerodynamic and acoustic fields of a rectangular supersonic jet," AIAA paper 2017-0002, 2017.

[36] Oertel, H., "Coherent structures producing Mach waves inside and outside of the supersonic jet," ISL-Report CO 218/82, 1982.

[37] Dimotakis, P. E., “Two-dimensional shear-layer entrainment,” AIAA Journal, Vol. 24, No. 11, 1986, pp. $1791-1796$. 